
\title{
Native Oxide Removal from InAIN Surfaces by Hydrofluoric Acid Based Treatment
}

\author{
Takuma NAKANO $^{\dagger \text { a) }}$, Nonmember and Masamichi AKAZAWA ${ }^{\dagger}$, Member
}

\begin{abstract}
SUMMARY We investigated the effects of chemical treatments for removing native oxide layers on InAlN surfaces by $\mathrm{X}$-ray photoelectron spectroscopy (XPS). The untreated surface of the air exposed InAlN layer was covered with the native oxide layer mainly composed of hydroxides. Hydrochloric acid treatment and ammonium hydroxide treatment were not efficient for removing the native oxide layer even after immersion for $15 \mathrm{~min}$, while hydrofluoric acid (HF) treatment led to a removal in a short treatment time of $1 \mathrm{~min}$. After the HF treatment, the surface was prevented from reoxidation in air for $1 \mathrm{~h}$. We also found that the 5 -min buffered HF treatment had almost the same effect as the 1-min HF treatment. Finally, an attempt was made to apply the HF-based treatment to the metal-InAlN contact to confirm the XPS results.

key words: InAlN, XPS, surface treatment, $H F$, native oxide
\end{abstract}

\section{Introduction}

InAlN lattice-matched to GaN has a large band gap, a large spontaneous polarization, and a large conduction band discontinuity at the InAlN/GaN interface. Owing to these features, a high density two-dimensional electron gas can be induced at the lattice-matched InAlN/GaN heterointerface [1]-[3]. Therefore, it has been proposed that highspeed, high-frequency, high-output, and high-efficiency devices can be obtained by using InAlN/GaN heterostructures [2]. Actually, field effect transistors with excellent performance characteristics have been achieved [3]-[5]. However, since research on InAIN has started only recently, the characteristics of and the control method for the surfaces and interfaces of InAIN have not been fully investigated.

After the epitaxial growth, it is necessary to expose the InAlN surface to air. Consequently, the formation of a surface native oxide layer is unavoidable, which possibly results in the generation of surface or interface states that affect the performance of electronic devices. Therefore, understanding the properties of the native oxide and establishment of control methods are important. On the basis of an X-ray photoelectron spectroscopy (XPS) study supported by electrical measurement, we investigated the effect of hydrofluoric acid (HF) based treatment for removing native oxide layer of InAIN.

\section{Experimental}

For XPS measurement, a 15-nm-thick undoped InAlN layer

\footnotetext{
Manuscript received August 25, 2012.

${ }^{\dagger}$ The authors are with Research Center for Integrated Quantum Electronics, Hokkaido University, Sapporo-shi, 060-8628 Japan. a)E-mail: tnakano@ rciqe.hokudai.ac.jp DOI: 10.1587/transele.E96.C.686
}

on a $\mathrm{GaN}$ buffer layer grown by metal organic vapor phase epitaxy (MOVPE) on a sapphire substrate was used for investigation. The same epitaxial wafer was cut into pieces and used for the investigation of chemical treatments using undiluted $\mathrm{HF}$ at room temperature (RT), buffered $\mathrm{HF}$ (BHF, HF: $\mathrm{NH}_{4} \mathrm{OH}=1: 5$ ) at RT, undiluted hydrochloric acid $(\mathrm{HCl})$ at $\mathrm{RT}$, and undiluted ammonium hydroxide $\left(\mathrm{NH}_{4} \mathrm{OH}\right)$ at $50^{\circ} \mathrm{C}$. The initial surface before the chemical treatments was that of air-exposed InAlN after growth. Prior to the chemical treatments, sample chips were cleaned with acetone. The chemical treatments were carried out by dipping the chips in each solution for 1 to $15 \mathrm{~min}$. Subsequently, the chips were rinsed with deionized (DI) water, blown with pure nitrogen and introduced into the analysis chamber immediately. DI water rinsing was necessary to prevent the contamination of the vacuum system by residual chemicals. XPS was performed using a monochromated Al- $K \alpha$ X-ray source $(1486.6 \mathrm{eV})$. The binding energy shift induced by the charging of the insulating substrate was calibrated by adjusting the peak position of the $\mathrm{C} 1$ s core level to $285.0 \mathrm{eV}$ for each sample surface. For electrical measurement, an $\mathrm{n}$-InAlN/n-AlN/n-GaN $(11 \mathrm{~nm} / 0.75 \mathrm{~nm} / 2 \mu \mathrm{m}$; all the layer were doped with Si up to $\mathrm{n}=1 \times 10^{18} \mathrm{~cm}^{-3}$ ) structure was also grown by MOVPE.

\section{Results and Discussion}

Figure 1 shows the core level spectral intensity ratio of $\mathrm{O} 1 \mathrm{~s}$ to $\mathrm{N} 1 \mathrm{~s},[\mathrm{O} 1 \mathrm{~s}] /[\mathrm{N} 1 \mathrm{~s}]$, for each treatment versus treatment time, with the result of the untreated sample for compari-

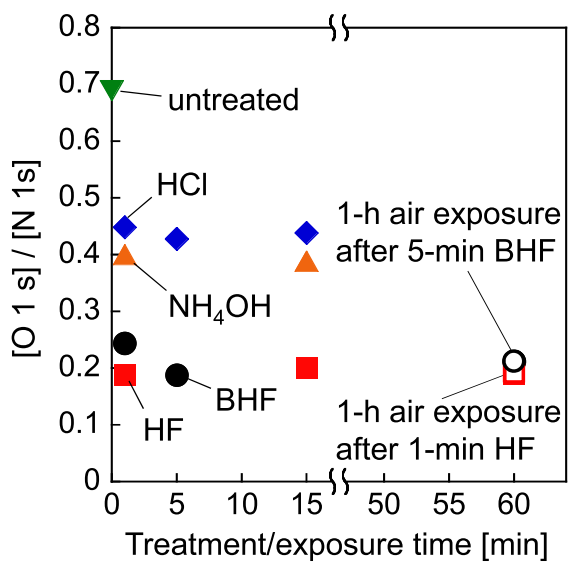

Fig. $1[\mathrm{O} 1 \mathrm{~s}] /[\mathrm{N} 1 \mathrm{~s}]$ vs treatment time or exposure time. 


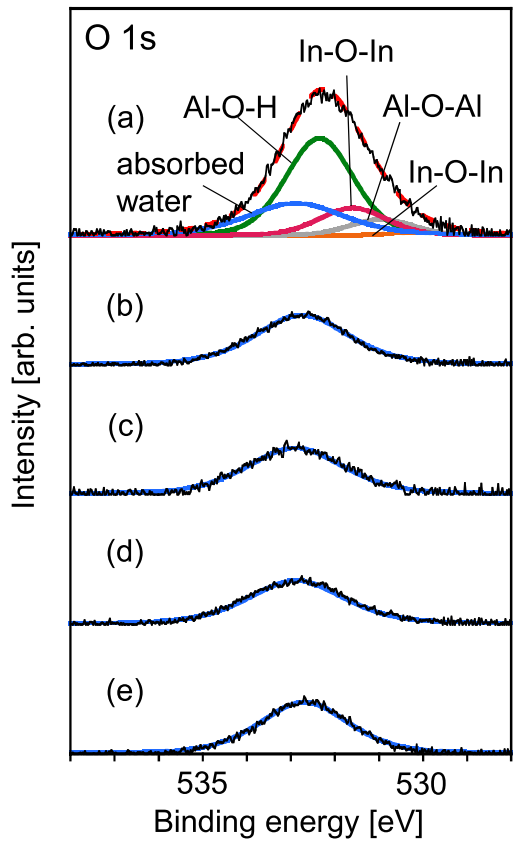

Fig. 2 O1s spectra obtained for (a) untreated sample and samples treated with (b) HF for $1 \mathrm{~min}$, (c) 1-h air exposure after 1-min HF treatment, (d) BHF for 5 min, and (e) 1-h air exposure after 5-min BHF treatment.

son. The HF treatment achieved the lowest intensity ratio among the three treatments, which was almost independent of treatment time. On the other hand, the $\mathrm{HCl}$ and $\mathrm{NH}_{4} \mathrm{OH}$ treatments exhibited higher intensity ratios even after a long treatment time of $15 \mathrm{~min}$. It can be seen that the HF treatment achieved the most efficient oxide removal within a treatment time as short as $1 \mathrm{~min}$.

To clarify the native oxide composition, $\mathrm{O} 1 \mathrm{~s}$ spectra were investigated. The XPS result on the untreated surface is shown in Fig. 2(a). The component at $530.0 \mathrm{eV}$ was assigned to be the In-O-In bond [6], whereas the energy separation between the In-O-In and In-O-H bonds of $1.6 \mathrm{eV}$ was consistent with the reported value between the main components of $\mathrm{In}_{2} \mathrm{O}_{3}$ and $\mathrm{In}(\mathrm{OH})_{3}$ [7], [8]. The component at $531.0 \mathrm{eV}$ was attributed to the Al-O-Al bond according to the measurement on sapphire substrates in good agreement with previously reported values [9], whereas the energy separation between the $\mathrm{Al}-\mathrm{O}-\mathrm{Al}$ and $\mathrm{Al}-\mathrm{O}-\mathrm{H}$ bonds of $1.4 \mathrm{eV}$ was in accordance with the previous report [9]. The component at $532.9 \mathrm{eV}$ was attributedd to the absorbed water component [7]. Oxynitride components are reported to be produced at temperatures of as high as $700^{\circ} \mathrm{C}$ [10]. Actually, there were no signs of oxidation in $\mathrm{N} 1 \mathrm{~s}$ spectra for the present sample.

XPS result indicated that the native oxide layer was mainly composed of $\mathrm{Al}(\mathrm{OH})_{3}$ and $\operatorname{In}(\mathrm{OH})_{3}$, which is consistent with the previously reported results for AlN [10] and InN [7]. The existence of a hydroxide layer on the surface of InAlN is unfavorable for device fabrication involving interface formation with other materials because the hydroxide is chemically and thermally unstable [7], [9]-[11]. Therefore, this layer should be removed prior to interface formation.

We found that all native oxide components were removed after the HF treatment for $1 \mathrm{~min}$ as shown in Fig. 2(b). The O 1s spectrum for the HF-treated sample was a single symmetry peak at $532.9 \mathrm{eV}$. In addition, the shape, intensity, and energy position of the $\mathrm{O} 1 \mathrm{~s}$ spectra after the same HF treatment were almost identical for the InAlN layer and a gold foil. Therefore, the $\mathrm{O} 1 \mathrm{~s}$ spectrum of the HF-treated InAlN was mainly assigned to the absorbed water molecules, although the absorbed organic molecules may have partially contributed to the spectrum. Consequently, a treatment time as short as 1 min was sufficient for removing the native oxide layer.

Even though the native oxide layer is removed by HF treatment, air exposure after the chemical treatment is unavoidable during a device fabrication process. If the HFtreated surface is reoxidized during the subsequent air exposure in a short time period, the treatment is meaningless. Considering the actual device fabrication process, the prevention of surface oxidation in air for $1 \mathrm{~h}$ after chemical treatment is sought. We checked this for the 1-min-HFtreated sample and found that the $[\mathrm{O} 1 \mathrm{~s}] /[\mathrm{N} 1 \mathrm{~s}]$ ratio did not increase with air exposure for $1 \mathrm{~h}$ as shown in Fig. 1. The $\mathrm{O}$ $1 \mathrm{~s}$ spectrum of the sample exposed to air for $1 \mathrm{~h}$ after the 1min HF treatment is shown in Fig. 2(c). No marked change was observed between this spectrum and that obtained immediately after the HF treatment shown in Fig. 2(b) including only the absorbed water component. Indeed, no sign of oxidation was observed in the In $3 \mathrm{~d}, \mathrm{~N} 1 \mathrm{~s}$, and $\mathrm{Al} 2 \mathrm{p}$ core level spectra as summarized in Fig. 3, where each spectrum obtained immediately after the HF treatment is also overlapped to clarify the congruity of the spectral shape. Consequently, the oxidation in air was prevented for $1 \mathrm{~h}$ after the HF treatment. Thus, we can conclude that the HF treatment for a short time of $1 \mathrm{~min}$ removes the InAlN native oxide layer and prevents reoxidation in air for a time period sufficient for a device fabrication process.

Although the superiority of the HF treatment in removing InAlN surface native oxide is evident, HF is characterized by a strong reactivity, to peel off the photoresist layer, and a high risk as a chemical solution. Therefore, BHF is usually used in actual device processing. Although the BHF treatment for $1 \mathrm{~min}$ resulted in a slightly higher $[\mathrm{O} 1 \mathrm{~s}] /[\mathrm{N} 1 \mathrm{~s}]$ ratio than the $\mathrm{HF}$ treatment, the BHF treatment for $5 \mathrm{~min}$ achieved an intensity ratio almost equal to that of the HF treatment as shown in Fig. 1. The $\mathrm{O}$ 1s spectrum for the BHF treatment is also shown in Fig. 2(d); this spectrum is almost identical to that of the HF-treated sample. This situation was maintained even after 1-h air exposure as shown Fig. 2(e). Thus, the BHF treatment can achieve the same effect as the HF treatment, though the suitable treatment time for oxide removal should be changed depending on the mixture ratio between $\mathrm{HF}$ and $\mathrm{NH}_{4} \mathrm{~F}$.

To confirm the effect of the oxide removal, HFbased treatment was applied to the fabrication process of metal-semiconductor junction diodes. The test diode structures were fabricated by forming $\mathrm{Ti} / \mathrm{Al} / \mathrm{Ti} / \mathrm{Au}$ 

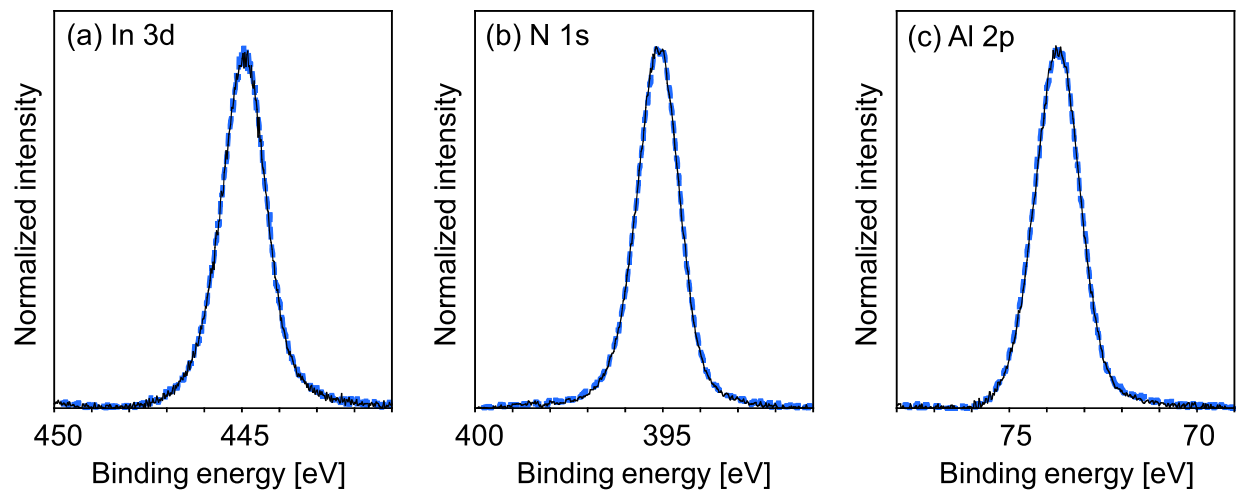

Fig. 3 (a) In 3d, (b) N 1s, and (c) Al 2p spectra. Solid lines indicate the spectra obtained after air exposure for $1 \mathrm{~h}$ after $1 \mathrm{~min}$ of $\mathrm{HF}$ treatment, while broken lines indicate the spectra obtained immediately after the HF treatment.

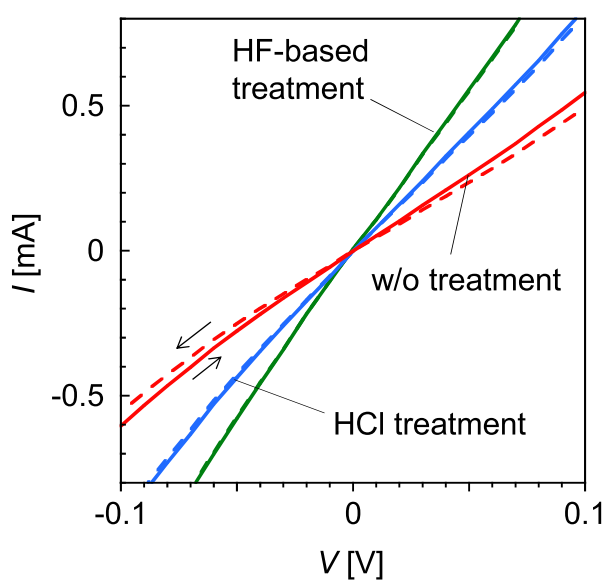

Fig. 4 I-V characteristics for the test diodes with the HF-based treatment, with the $\mathrm{HCl}$ treatment and without treatment. Though the bias voltage was applied from $-3 \mathrm{~V}$ to $3 \mathrm{~V}$ for solid lines and from $3 \mathrm{~V}$ to $-3 \mathrm{~V}$ for broken lines, this is enlarged plot to clarify the hysteresis.

$(20 \mathrm{~nm} / 50 \mathrm{~nm} / 20 \mathrm{~nm} / 100 \mathrm{~nm})$ electrodes onto untreated and chemically treated surfaces of the $n-I n A l N / n-A l N / n-G a N$ structures. The electrode pattern consisted of a $260-\mu \mathrm{m}-$ diameter circle in a sufficiently large surrounding electrode via a $20-\mu \mathrm{m}$-width annular gap. Here, HF-based treatment consisted of HF treatment before the photolithography process and BHF treatment prior to metal deposition after photolithography. For comparison, untreated and $\mathrm{HCl}$ treated samples were also prepared. $\mathrm{HCl}$ treatment was applied before and after photolithography. The current-voltage (IV) characteristics were measured laterally between circular and surrounding $\mathrm{Ti} / \mathrm{Al} / \mathrm{Ti} / \mathrm{Au}$ electrodes on the InAlN surface. Unlike the $\mathrm{AlGaN} / \mathrm{GaN}$ structures with the similar doping density [12], complete nonalloyed ohmic contacts were not obtained presumably owing to the higher barrier of InAlN. Still, as shown in Fig. 4, the I-V characteristic for the HF-treated sample indicated the largest current, while untreated and $\mathrm{HCl}$-treated samples showed higher resistance. It should be noted that the HCl-treated sample showed intermediate characteristic between HF-treated and untreated samples. In addition, hysteresis was minimized by the HF- based treatment, and was the largest for the untreated sample. $\mathrm{HCl}$ treatment led to an intermediate magnitude, again. Therefore, it was highly likely that the native oxide layer at the metal-semiconductor interface dominated the hysteresis. These results supported the XPS results.

\section{Conclusion}

The effects of chemical treatments for removing native oxide layers on InAlN surfaces were investigated by XPS. The untreated surface of the air exposed InAlN layer was covered with the native oxide layer mainly composed of hydroxides. The $\mathrm{HCl}$ and $\mathrm{NH}_{4} \mathrm{OH}$ treatments were not efficient for removing the native oxide layer even after immersion for $15 \mathrm{~min}$, while the HF treatment led to a removal in a short treatment time of $1 \mathrm{~min}$. After the HF treatment, the surface was prevented from reoxidation in air for $1 \mathrm{~h}$. We also found that the 5-min BHF treatment had almost the same effect as the 1-min HF treatment. Finally, the HFbased treatment was applied to the metal-InAlN contact to confirm the XPS results.

\section{Acknowledgments}

This work was supported by JSPS KAKENHI Grant Number 24560022. The authors thank Prof. T. Hashizume and Prof. E. Sano, Hokkaido University, and Dr. M. Hiroki, NTT Photonics Laboratories, for their fruitful discussions.

\section{References}

[1] R. Ambacher, M. Dimitrov, B.E. Stutzmann, M.J. Foutz, J.A. Murphy, J.R. Smart, N.G. Shealy, K. Weimann, M. Chu, B. Chumbes, A.J. Green, W.J. Sierakowski, Schaff, and L.F. Eastman, "Role of spontaneous and piezoelectric polarization induced effects in gourp-III nitride based heterostructures and devices," Phys. Stat. Sol. (B), vol.216, pp.381-389, 1999.

[2] J. Kuzmík, "Power electronics on InAlN/(In)GaN: Prospect for a record performance," IEEE Electron. Device Lett., vol.22, no.11, pp.510-512, 2001.

[3] M. Higashiwaki and T. Matsui, "InAlN/GaN heterostructure fieldeffect transistors grown by plasma-assisted molecular-beam epitaxy," Jpn. J. Appl. Phys., vol.43, no.6B, pp.L768-L770, 2004. 
[4] M. Hiroki, N. Maeda, and N. Shigekawa, "Compressively strained $\mathrm{In}_{\mathrm{x}} \mathrm{Al}_{1-\mathrm{x}} \mathrm{N} / \mathrm{Al}_{0.22} \mathrm{Ga}_{0.78} \mathrm{~N} / \mathrm{GaN}(\mathrm{x}=0.245-0.325)$ heterostructure field effect transistors with regrown AlGaN contact layers," Jpn. J. Appl. Phys., vol.49, 04DF13, 2010.

[5] D.S. Lee, J.W. Chung, H. Wang, X. Gao, S. Guo, P. Fay, and T. Palacios, "245-GHz InAlN/GaN HEMTs with oxygen plasma treatment," IEEE Electron. Device Lett., vol.32, no.6, pp.755-757, 2011.

[6] C. Nunes de Carvalho, A.M. Botelho do Rego, A. Amaral, P. Brogueira, and G. Lavareda, "Effect of substrate temperature on the surface structure, composition and morphology of indium-tin oxide films," Surf. Coatings Technol., vol.124, pp.70-75, 2000.

[7] N. Asai, Y. Inoue, H. Sugimura, and O. Takai, "Electrochromic reaction of InN thin films," J. Electrochem. Soc., vol.146, no.6, pp.23652369, 1999.

[8] G. Hollinger, E. Bergignat, J. Joseph, and Y. Robach, "On the nature of oxides on InP surfaces," J. Vac. Sci. Technol. A, vol.3, no.6, pp.2082-2088, 1985

[9] M.R. Alexander, G.E. Thompson, and G. Beamson, "Characterization of the oxide/hydroxide surface of aluminium using X-ray photoelectron spectroscopy: A procedure for curve fitting the $\mathrm{O} 1 \mathrm{~s}$ core level,” Surf. Interface Anal., vol.29, pp.468-477, 2000.

[10] A.D. Katonani and K.I. Papathomas, "Kinetics and initial stages of oxidation of aluminum nitride: Thermogravimetric analysis and $\mathrm{X}$ ray photoelectron spectroscopy study," J. Vac. Sci. Technol. A, vol.5, no.4, pp.1335-1340, 1987.

[11] H.M. Liao, R.N. S. Sodhi, and T.W. Coyle, "Surface composition of AIN powders studied by X-ray photoelectron spectroscopy and bremsstrahlung-excited auger electron spectroscopy," J. Vac. Sci. Technol. A, vol.11, no.5, pp.2681-2686, 1993.

[12] J.S. Jang, T.Y. Seong, and S.R. Jeon, "Electronic transport mechanism for nonalloyed Ti-based Ohmic contacts to n-AlGaN," J. Appl Phys., vol.100, no.4, 046106, 2006. 